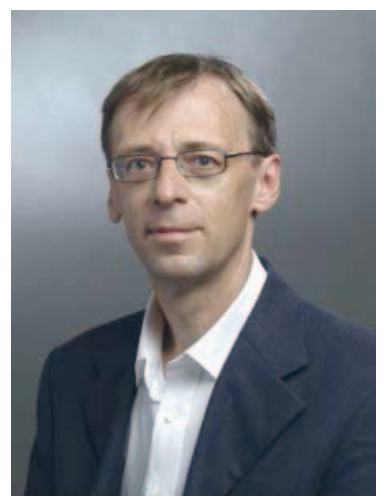

Dr. med. Martin Frei-Erb, Bern, Schweiz

\section{Vom Hausärztemangel ist auch die Komplementärmedizin betroffen}

Der Mangel an Hausärzten in europäischen Ländern wird zunehmend gravierender. In der Schweiz werden bis 2016 $50 \%$ der praktizierenden Hausärzte in Pension gehen. Nachfolger für diese 3200 Grundversorger sind kaum in Sicht, Lösungen noch weniger, obwohl alle Gesundheitspolitiker beeindruckt sein sollten, dass Hausärzte 90\% der gesundheitlichen Probleme ihrer Patienten zu 7\% der Gesundheitskosten von aktuell ca. 58 Milliarden CHF erfolgreich lösen [1]. Vergessen wird dabei, dass diese Leistung ohne Einbezug "unkonventioneller» Behandlungsmethoden nicht möglich wäre [2]. Betroffen von dieser existenzbedrohenden Situation der ärztlichen Grundversorgung sind damit auch viele komplementärmedizinische Ärzte.

Die Gründe für das Desinteresse der Studierenden der Medizin an einer Weiterbildung zum Grundversorger nur in den langen Arbeitszeiten, häufigen Notfalldiensten auch an Wochenenden, der im Vergleich zu Spezialärzten mangelhaften finanziellen Entschädigung oder gar bei den Ärztinnen («Feminisierung der Medizin») zu suchen, ist aus meiner Sicht zu oberflächlich.

Nachdenklich stimmt eine Pilotstudie bei Studierenden der Universitäten Bern und Lausanne, in der die vorhandenen Kompetenzen der Teilnehmenden in verschiedenen medizinischen Teilbereichen mit den von Experten in Workshops erarbeitenden Soll-Kompetenzen verglichen worden sind. Die Mehrzahl der Studierenden verfügt über die notwendigen Kompetenzen in den patientenfernen Bereichen Forschung und Verwaltung, hat aber mangel- hafte Kompetenzen als Haus- oder Spitalarzt [3]. Offenbar werden bereits bei der Selektion und Ausbildung der Studierenden grundlegende Kompetenzen der ärztlichen Kunst zu wenig berücksichtigt.

Über die Ursachen dafür kann nur spekuliert werden. Nicht von der Hand zu weisen ist allerdings der Drang der universitären Medizin, wo mehrheitlich die Ausbildung unseres Nachwuchses erfolgt, sich als reine Naturwissenschaft zu präsentieren. Diagnosen wie «AML mit inv(16)(p13.1q22)/t(16;16)(p13.1;q22), CBFB-MYH11» drohen wichtiger zu werden als Empathie und die Akzeptanz unserer Patienten als Individuen mit subjektiven Wertvorstellungen und Krankheitskonzepten auf dem Hintergrund ihrer Lebens- und Krankheitsgeschichte. Diese Tendenz führt die Medizin weg von einer praktischen Wissenschaft, vom «Know-how», zu einer theoretischen Wissenschaft, zum «Know-that». Studierende, die auf diese Weise ausgebildet werden, «wissen» mehr, «können» aber weniger und sind damit eben in patientenfernen Bereichen kompetenter.

Aus dem Verständnis einer Krankheit als zu reparierende Störung eines Körperorgans oder als genetischer Defekt und dem blinden Vertrauen in wissenschaftliche Untersuchungen erfolgt oft die unkorrekte Anwendung der evidenzbasierten Medizin mit lediglich Berücksichtigung der externen Evidenz, aber ohne Einbezug der Präferenzen der Patienten und der klinischen Erfahrung, d.h. des Könnens des behandelnden Arztes [4]. Derartige Fehlleistungen in der Ausbildung von zukünftigen Ärzten sind unter anderem für das schlechte Image der Hausarzt- und Komplementärmedizin als «unwissenschaftliche» Fachrichtungen verantwortlich. Wer nichts weiss, wird Hausarzt, oder noch schlimmer: «Alternativmediziner».

Dabei warten in der Grundversorgung grosse und zugleich spannende Herausforderungen: Die gestiegene Lebenserwartung führt dazu, dass wir in unseren Arztpraxen immer mehr Patienten mit chronischen Krankheiten behandeln. Evidenzbasierte Medizin und Guidelines sind dabei selten von Nutzen, da diese Patienten oft komplexe Krankheitsbilder mit einer individuellen Mischung aus körperlichen, psychischen und sozialen Problemen zeigen [5]. Mit der Schlussfolgerung der von Kamerow zitierten Arbeitsgruppe aus Kanada, dass nur Grundversorger in der Lage sind, die Behandlung multimorbider Patienten zu übernehmen und zu koordinieren, und dabei partnerschaftliche, patientenzentrierte und zielorientierte

\section{KARGER}

Fax +497614520714 Information@Karger.com www.karger.com
Dr. med. Martin Frei-Erb

Kollegiale Instanz für Komplementärmedizin KIKOM

Universität Bern, Inselspital, Imhoof-Pavillon

3010 Bern, Schweiz

martin.frei@kikom.unibe.ch 
Behandlungsmodelle vorzuziehen sind, schliesst sich der Kreis zur Komplementärmedizin. Ärzte mit einer Zusatzqualifikation in einer komplementärmedizinischen Methode sind aufgrund des ganzheitlichen Krankheitsverständnisses Experten für komplexe Krankheitsbilder.

Wie sollen wir Komplementärmediziner nun mit dieser Diskrepanz zwischen steigender Patientennachfrage und fehlenden Ärzten umgehen? Als praktizierende Ärzte können wir weder auf die Ausbildung der Studierenden an den Universitäten noch auf die gesetzlichen Vorgaben zu Tarif, Notfalldienst usw. gross Einfluss nehmen, was ein Gefühl der Ohnmacht und Resignation und damit einen Rückzug in eine (komplementär-)medizinische Parallelwelt auslösen kann. Nachhaltiger scheint mir persönlich, dass wir unsere Expertise nicht nur unseren Patienten zugutekommen lassen, sondern diese auch aktiv unseren konventionell-medizinischen Kollegen, und hier vor allem den Studierenden und jungen Kollegen in Weiterbildung, zur Verfügung stellen.

Zweckmässiger und wirksamer als belehrende Broschüren und Informationsveranstaltungen mit einseitiger Darstellung der Vorteile der Komplementärmedizin und der Nachteile der Schulmedizin, wie dies leider in der Vergangenheit oft geschehen ist, sind dabei persönliche Kontakte und die sich daraus ergebenden Gespräche und Diskussionen. Dies erfordert persönliche Präsenz und Engagement, beginnend bei lokalen und regionalen Veranstaltungen bis zu nationalen und internationalen Kongressen, sowie gegenseitige Toleranz und Respekt vor den Stärken und Schwächen der jeweils «anderen» Medizin. Eminent wichtig ist die Beteiligung am studentischen Unterricht, soweit dies möglich ist, und das Angebot von Praxisassistenzstellen, deren Finanzierung immer häufiger durch öffentliche Gelder unterstützt wird. Aus Erfahrung wissen wir alle, dass Vorbilder und eindrücklich miterlebte Krankheitsverläufe für unseren Lernprozess prägender sind als das Anhäufen von theoretischen Informationen.

Und zu guter Letzt sollten wir es nicht verpassen, unsere jungen Kollegen immer wieder darauf aufmerksam zu machen, dass Grundversorger mit einer Zusatzqualifikation in Komplementärmedizin nicht nur zufriedenere Patienten haben, sondern selber auch eine höhere Berufszufriedenheit aufweisen [6].

Es liegt nun in unserer Hand, nicht einfach zu akzeptieren, dass wir keine Nachfolger finden werden, sondern die vielen positiven Aspekte unserer beruflichen Tätigkeit grossflächig in der medizinischen Welt zu verbreiten. Unsere Patienten werden uns dafür dankbar sein.

\section{Literatur}

1 www.hausaerzteschweiz.ch/hausarztmedizin.

2 Déglon-Fischer A, Barth J, Ausfeld-Hafter B: Komplementärmedizin in Schweizer Praxen der Grundversorgung. Forsch Komplementmed 2009;16:251-255.

3 Menzi B, Weber N: In der Humanmedizin sind künftig neue Kompetenzen gefragt. Schweiz Ärztez 2012;93:1564-1566.

4 Sackett D, Rosenberg W, Gray J, Haynes R: Evidence based medicine: what it is and what it isn't. BMJ 1996;312:71-72.

5 Kamerow D: How can we treat multiple chronic conditions? BMJ 2012;344:10-11.

6 Joos S, Musselmann B, Szecsenyi J, Goetz K: Characteristics and job satisfaction of general practitioners using complementary and alternative medicine in Germany - is there a pattern? BMC Complement Altern Med 2011;11:131. 